\title{
GW23-e0570 COMPARATIVE STUDY OF TWO OCCLUDER-RELEASE TECHNIQUES IN TRANSCATHETER CLOSURE OF PERIMEMBRANOUS VENTRICULAR SEPTAL DEFECT
}

doi:10.1136/heartjnl-2012-302920p.7

Zhou Aiqin, Zhong Yiming, Xia Dongming, Liu Hailong, Yang Yihong, Zhou Aiqin. The First Affiliated Hospital, Gannan Medical University, Ganzhou, China

Objectives Reported experience with the retained guidewire technique in transcatheter closure of perimembranous ventricular septal defect (VSD) is limited. To compare two occluder-release techniques in transcatheter closure of perimembranous VSD we reviewed our experience in 48 procedures performed on 48 patients.

Methods From January 2009 to January 2011, 48 patients (26 males and 22 females) with perimembranous VSD underwent an attempt of transcatheter closure using the amplatzer occluder

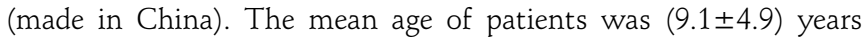
(ranged from 3 to 18 years). The mean body weight of patients was $(31.1 \pm 17.9) \mathrm{kg}$ (ranged from 12 to $52 \mathrm{~kg}$ ). The mean diameter of VSD measured by transthoracic echocardiography (TTE) was $(6.4 \pm 3.6) \mathrm{mm}$ (ranged from 3 to $11 \mathrm{~mm}$ ). The patients were grouped by either the retained guidewire technique $(n=24)$ or non-retained guidewire technique $(n=24)$. Occluder was released through the right heart system. During the operation, an arteriovenous guide wire loop with a 0.035 inch exchange length guide wire (Cordis) was set up through a femoral vein approach. A long sheath was advanced to the left ventricle through the arteriovenous guide wire loop and positioned beneath the aortic valve. Then the guide wire was removed traditionally in non-retained guide wire group, or was not removed creatively in retained guide wire group. The VSD occluder was deployed through the long sheath under fluoroscopic control and echo cardiographic guidance. Procedure time, X-ray exposing time, technical successful rate and complication rate were compared between two groups. All patients were followed up in 1, 6 and 12 months after procedures of TTE, X-ray and electrocardiography.

Results No significant differences were found in gender, age, weight, the average diameter of VSD $(6.3 \pm 3.5$ vs $6.5 \pm 3.7 \mathrm{~mm}$, $\mathrm{p}>0.05)$, the distance from VSD to aortic valve $(2.3 \pm 1.9$ vs 2.5 $\pm 2.1 \mathrm{~mm}, \mathrm{p}>0.05)$ or the average diameter of amplatzer occluder $(9.5 \pm 4.1$ vs $9.7 \pm 5.2 \mathrm{~mm}, \mathrm{p}>0.05)$. No significant difference was found in technical successful rate $(91.7 \%$ vs $95.8 \%, p>0.05)$, number of applications, or major complication rate $(2.1 \%$ vs $2.1 \%$, $\mathrm{p}>0.05)$. The procedure time in retained guidewire group was less than that in non-retained guide wire group $(66.2 \pm 31.4$ vs 97.5 $\pm 54.5 \mathrm{~min}, \mathrm{p}<0.05)$. The $\mathrm{X}$-ray exposing time in retained guide wire group was less than that in non-retained guide wire group $(12.4 \pm 6.3$ vs $21.6 \pm 10.7$ min, $p<0.05)$. Except 7 cases with mild residual shunt that disappeared in 12 months, no serious complication were found in the other patients, such as device transposition, haemolysis, atrioventricular block, valvular regurgitation and cardiac perforation.

Conclusions These data suggest that although outcomes and major complication rates are similar for the two groups, transcatheter closure of perimembranous ventricular septal defect with retained guide wire technique would shorter operative time, and reduce the amount of x-ray irradiation of the doctors and patients. 